\title{
Route of Administration of Corticosteroids and Asthma Relapse after Acute Care; Appraisal of Evidence on Effectiveness and Safety
}

\author{
Sogunle P.T ${ }^{1 *}$, Sogunle E.O ${ }^{2}$ \\ ${ }^{1}$ Department of Family Medicine, Federal Medical Centre, Abeokuta \\ ${ }^{2}$ Department of Public Health, Witwaterstrand University, South Africa
}

\begin{abstract}
Patients managed for acute exacerbation of asthma in acute settings respond to standard management protocol involving systemic and oral steroids. However, relapses are common due to poor adherence to tapering doses of oral steroids. Intramuscular (IM) administration of corticosteroids has been shown to be equipotent to oral steroids with the added advantage of convenient dosing and improved adherence. However, the role of the route of administration of corticosteroids related to relapse of asthma attacks after acute care has not been fully explored. To find and appraise up-to-date evidence in the literature that compares the effectiveness and safety of IM corticosteroids o a shortcourse oral corticosteroid in the treatment of asthma patients in acute care settings. The principles of evidence-based medicine (EBM) were used. Suitable articles were identified using MEDLINE, Cochrane library, and Google scholar. Titles and summary texts were reviewed. The full article of the relevant article was downloaded for critical appraisal of methodological quality using a measurement tool to assess systematic review 2 (AMSTAR 2). In applying AMSTAR 2 to the selected systematic review article, it was noted that the investigators followed most of the requirements. However, adequate investigation of publication bias and discussion of its likely impact on the review's result was not done by the researchers. No significant differences in effectiveness and safety were observed between IM corticosteroids and oral corticosteroids among this category of patients. Additional studies are required to compare different IM and oral corticosteroid preparations.
\end{abstract}

Keywords: Acute exacerbation of Asthma, AMSTER 2, Evidence-based medicine, Route of administration of corticosteroids.

\section{Introduction}

The clinical features of asthma resulting from reversible airway inflammation include chest tightness, worsening cough, shortness of breath, and wheezing [1]. In our context, most patients managed for acute exacerbation of asthma respond favourably to treatment with standard management protocol [2]. Systemic and oral steroids are administered, followed by gradual resolution of symptoms. Parenteral corticosteroids are ideally converted into oral steroids. Upon discharge, most patients have prescribed a tapering regimen of oral steroids in addition to inhaled steroid preparations using metered-dose inhalers. However, the rate of relapse requiring admission for further treatment is common. In literature, reports of relapse of acute asthma after the patient has been discharged from acute care settings ranges between 10 and 31\% [3-5]. This situation in this category of patients constitutes a need for a review of asthma management protocol in acute care settings.

In the reduction of the relapse rate of asthma following acute care, sufficient evidence has not been provided to support the role of the route of administering corticosteroids. Suggested associations have been reported with corticosteroids (particularly those with a depot- 
repository release) given to asthmatic patients before discharge from an emergency department via the intramuscular (IM) route [6-10] Intramuscular repository corticosteroids were found to be as equally useful as oral corticosteroids in these studies. It has been observed that the main challenge with asthma patients taking oral steroids in tapering dose is adherence. As a result of the high number of drugs and regular dosage changes, dosage tapering of oral steroids has been found to be complicated. As such, there is a lack of adherence after discharge in about $12 \%-22 \%$ of these patient $[11,12]$. This situation may be contributory to the relapse of acute exacerbation of asthma after acute care.

We, therefore, sought to find and appraise current research on asthma patients in acute care settings (emergency department or equivalent) that compares the effectiveness and safety of IM corticosteroids to oral corticosteroids before and after discharge, respectively. We made use of the principles of evidence-based medicine (EBM) for this purpose. We planned to search for up-todate evidence and critically appraise the evidence with an appropriate tool to determine the validity and utility of this evidence in this context. This review involves the first three steps in the recommended five general steps of EBM. We hope this paper will generate the needed evidence to carry out the remaining two steps in EBM.

\section{Methods}

\section{Evidence-Based Medicine}

The 5 steps in the EBM model are [13];

1. Identification of clinical question.

2. Best or suitable evidence.

3. Evidence critical appraisal for usefulness and validity.

4. Evidence results in application in clinical practice.

5. Evaluation of skill, information, and EBM procedures.

Evidence-based medicine can be defined as a systematic approach to solving clinical problems by integrating superlative research evidence with patient values and clinical expertise. It refers to judicious, conscientious, and explicit use of up-to-date superlative evidence in making appropriate clinical decisions [14-16]. The EBM tools include; identifying, critically appraising, and incorporation of evidence results into daily clinical practice. Searching for current best evidence is vital, and randomized controlled trials (RCTs) are mostly preferred for interventions [16]. Systematic reviews of RCTs in applied health research have become the standard method used in assessment and summarization $[17,18]$. The results obtained are of the highest level when compared to other research designs [19].

\section{Step 1: Generation of an Answerable Clinical Question}

This is the first and most important step in EBM. An important challenge in this step is to formulate an unanswerable clinical question [20]. A good question should be relevant and specific; communicated distinctly; With a clear objective and of necessity; and time-bound [19].

With regards to the interest of our article, using the PICO format, 4 or 3 of the items listed below must be included in a good clinical question:

1. Problem or patient: Patients with frequent relapse in attacks of asthma following discharge from emergency departments or acute care settings.

2. Intervention: IM corticosteroids.

3. Comparison: Oral Steroids.

4. Outcomes: Effectiveness and safety in the reduction in the incidence of relapse of asthma attacks after patients have been discharged from the acute care setting or its equivalent.

\section{A Four-Part Clinical Question}

In patients with recurrent relapses in attacks of asthma following discharge from acute care, are IM corticosteroids more effective and safer in the reduction of the incidence of relapse of 
asthma attacks when compared with oral steroids?

\section{Step 2: Finding the Evidence}

To identify previously published information to answer the research question, online electronic bibliographic databases were searched. It is important in EBM to use these databases effectively. MEDLINE, Cochrane library, and Google scholar were selected for use in this present article. The search terms used in the selected databases were: asthma, relapse, recurrence, emergency, acute care (problem P), IM corticosteroids, triamcinolone, depo repository steroid, dexamethasone, prednisolone (intervention I), oral steroid, oral steroid administration, (comparison $\mathrm{C}$ ), effectiveness, safety (outcome $\mathrm{O}$ ), randomized controlled trials, clinical trials, systematic review, metaanalysis (study design SD). We searched for a systematic review of randomized controlled studies or randomized controlled study, to provide information to answer the clinical question.

\section{Conducting the Search}

We commenced the search in PUBMED (MEDLINE) using certain keywords and generated terms on 20 July 2020 . We made use of the Boolean operators "AND" and "OR" to combine individual words or terms [21]. We used AND to retrieve articles containing all terms, while OR was used to retrieve articles containing either term. Five different search terms were used in MEDLINE resulted in 14 articles been highlighted (See supplementary list 1). The last $\left(6^{\text {th }}\right)$ search term combined the previous five searches with the AND Boolean operator, and 14 articles were retrieved. After reviewing the titles and summary texts of these fourteen articles, one article was found to be relevant. We did not search the Cochrane library because this article came from the Cochrane Database of Systematic Reviews. The full article of the only relevant article [22] was downloaded for critical appraisal of the quality of methodology. The AMSTAR 2 (A Measurement Tool to Assess Systematic Reviews 2) tool was used [23].

\section{Results}

\section{Article Selected}

Intramuscular versus oral corticosteroids to reduce relapses following discharge from the emergency department for acute asthma Kirkland SW, Cross E, Campbell S, Villa-Roel C, Rowe BH.

\section{Discussion}

\section{Step 3: AMSTAR Tool - Appraising the Evidence}

The quality of the methodology of systematic reviews can be assessed using the first AMSTAR, a measurement tool [24]. AMSTAR, an 11 - item widely used assessment tool developed for the evaluation of systematic review researches conducted for randomized trials. There is currently an increase in its use by authors, editors of journals, healthcare policymakers, as well as for assessments of agencies for health technology [24]. Multiple critiques of this tool have been published [22]. An updated version has recently been developed - AMSTAR 2 [23]. This version is believed to make decisions based on observational evidence in the real world, thereby assisting those making decisions in selecting systematic reviews of high quality.

The revised instrument (AMSTAR 2) has the following characteristics;

1. Overall - 16 items (10 items from the previous version).

2. The response categories are easier to follow than the first AMSTAR.

3. A comprehensible user manual is present.

4. Overall rating is present.

There are risks associated with accommodating the results obtained from one systematic review without a critical review of the quality of its methodology. Prior to AMSTAR, certain assessment tools have been developed i.e., Preferred Reporting Items for 
Systematic Reviews and Meta-Analyses (PRISMA) statement [24, 25] and Meta-analysis of Observational Studies in Epidemiology (MOOSE) [26, 27]. However, these tools do not focus on the way the authors conducted their review but rather how good the authors write in a comprehensible manner. Therefore, AMSTAR 2 as a measurement tool evaluates the methods used in planning and conducting a review [25]. The comprehensive AMSTAR 2 tool can be seen in supplementary list 2 [23].

\section{Application of AMSTAR 2 to the Systematic Review Article Selected.}

As strongly recommended by the developers, the combination of individual item ratings to add up to the total score was not done [23]. Instead, the effect of the possible inadequate rating for individual items were considered.

\section{On the Use of PICO for Research Questions and Inclusion Criteria for the Review}

PICO was used by the authors appropriately. Hence the research question reads, "To examine the effectiveness and safety of a single dose of intramuscular (IM) corticosteroids provided prior to discharge compared to a short course of oral corticosteroids in the treatment of acute asthma patients discharged from an ED or equivalent acute care setting."

\section{Presence of Explicit Statement from Review Protocol or Significant Deviation}

Yes - The review was conducted in line with the published protocol, and differences between the protocol and the review was discussed in a related section [22]. In this section, the authors highlighted the differences between the review and protocol. In response to peer review, the authors added an additional sensitivity analysis, in which studies that provided patients with additional corticosteroids as a co-intervention were excluded from the analysis. Risk of bias assessment was carried out on each included study.

\section{Selection of the Study Designs for Inclusion in the Review}

Yes - They selected RCTs or controlled trials conducted on paediatric or adult patients with acute asthma in an acute setting or equivalent. The studies compared the effectiveness of treatments with intramuscular or oral corticosteroids.

\section{Comprehensive Literature Search Strategy}

Yes - Most recently, on the 14th March 2018, searches were done on the Cochrane Airways Group Register of Trials. An extensive search was previously done in April 2017 - nine electronic databases were searched. They include Proquest Dissertations, Global Health, Embase, SCOPUS, Medline, LILACS, International Pharmaceutical Abstracts, EBMALL, CINAHL, and Theses Global. Grey literatures were searched. The list of references of review articles and primary studies and relevant manufacturers' websites were also searched. There was no restrictions on publication or language status. Twenty-four months or less was the time limit for the completion of the review of included literature.

\section{Duplication of Selected Study}

There was an independent screening of studies by two review authors (SWK and EC). Inclusion and exclusion criteria were identified. A third review author (CVR or BHR) was sometimes used during some disagreements. Duplicates were identified and excluded. The selection process was recorded in detail, and the PRISMA flow diagram was completed.

\section{Duplication of Data Extraction}

The authors used a data collection form for study characteristics and outcome data, which was piloted on at least one included study in the review. Two review authors (SWK and EC) independently extracted the following study characteristics into data collection forms from the included studies - Methods, Participants, Interventions, Outcomes Funding and notable 
conflict of interest of study authors shown in the 'Characteristics of included studies' table. Disagreements were resolved by reaching a consensus or through the involvement of a third review author (CVR or BHR). One of the review authors, SWK, used the Review Manager 5 (RevMan 5) file. To ensure adequate data entering, a second review author (EC) verified the extracted data for accuracy against the study report.

\section{List of Excluded Studies and Justification for Exclusions}

Yes - The full - text of twenty studies were downloaded by the authors for review. After this, eleven studies were excluded due to inappropriate design. The reasons for exclusion are presented in the table of characteristics for studies that were excluded in the article.

\section{Description of the Studies Included in Detail}

Yes - a total of nine studies were included. This was adequately shown on a table showing study designs, participants, intervention, comparators, outcome, and risk of bias.

\section{Usage of Satisfactory Technique to Assess the Risk of Bias (RoB) in Included Studies}

The risk of bias for each included study was examined using Cochrane's risk of bias tool. The domains examined were directed towards: (a) selection bias - allocation concealment \& random sequence generation; (b) Reporting bias - selective reporting; (c) Data attrition bias incomplete outcome; (d) Detection bias blinding of outcome assessment and other bias like a source of funding.

\section{Reported Sources of Funding for the Studies Included in the Review}

Yes - sources of funding for the review was reported by the authors. They include National Institute for Health Research (NIHR), via Cochrane Infrastructure funding to the Cochrane Airways Group; in-kind resources by the Emergency Medicine Research Group (EMeRG) affiliated with the Department of Emergency
Medicine University of Alberta; Canadian Institutes of Health Research (CIHR); CIHR in partnership with the Knowledge Translation branch, tier I Canada Research Chair in Evidence-based Emergency Medicine from CIHR through the Government of Canada (Ottawa, Ontario); Alberta Innovates Health Solutions (AIHS) Summer Studentship Award (Edmonton, Alberta).

\section{For Meta-analysis - Use of Appropriate Methods}

Yes, Meta-analysis was performed. Relative risk values, standardized mean difference or mean difference, were presented where appropriate.

For Meta-analysis - Assessment of the Potential Impact of RoB in Individual Studies on the Results

Yes, Meta-analysis was performed. In the summary of the findings' table, outcomes included all relapse i.e., relapse within or after ten days of discharge, adverse events, PEF/FEV, symptom scores, and beta-agonist use in a 24hour period. The five GRADE considerations (i.e. indirectness, risk of bias, publication bias, consistency of effect, and imprecision) were used to assess the quality of research for the outcome in question. The authors used the methods and recommendations described in Section 8.5 and Chapter 12 of the Cochrane Handbook for Systematic Reviews of Interventions [28, 29]. They used Grading of Recommendations Assessment, Development and Evaluation (GRADE) pro software. Footnotes were used to explain changes made to reduce the quality if studies were included. The risk of bias for the included studies was noted.

\section{Risk of Bias in Primary Studies during Discussion of the Results}

Yes. The authors included RCTs with a moderate or high risk of bias $(\mathrm{RoB})$. The total RoB ranged from unclear to high. None had a bias of low risk. Those (four) with a bias of high risk were not placebo-controlled trials. The other 
five studies had bias with unclear risk. In addition, the majority of studies did not adequately describe their method of randomization. Using GRADE, the total quality of the outcomes ranged from low to moderate. They were reduced to low quality, the primary outcomes of relapse as well as relapse after 10 days due to overall unclear to high risk of bias of the studies, and imprecision due to wide confidence intervals including both benefit, harm, and no effect.

The quality of the outcome adverse events was judged to be low quality due to the overall unclear to high risk of bias of the included studies and imprecision due to few events. They considered both 'symptom persistence' and '24hour beta-agonists use' to be low quality due the overall unclear to high risk of bias of the included studies, as well as few events. They assessed the 'outcome of relapse within 10 days' to be of moderate quality due to the overall unclear to high risk of bias of the studies. They reduced 'peak expiratory flow' to moderate quality due to imprecision of the results.

\section{Provision of Satisfactory Explanation for any Heterogeneity Observed}

Yes - The authors measured heterogeneity between the studies in the respective analysis. Significant heterogeneity was reported associated factors were examined using subgroup analysis. To examine sources of heterogeneity, the following analysis were conducted:

1. Children (zero to 18 years) versus adults (18 years \& older) to examine any potential agespecific treatment effects of IM or oral corticosteroids.

2. Relapse occurring within 10 days and over 10 days post-discharge.

3. Low versus moderate versus high exacerbation severity based on the pulmonary function obtained when a participants presented in the acute care setting or its equivalent prior to treatment with a bronchodilator.
4. Co-interventions received (ICS versus ICS corticosteroids/ Long-acting beta-agonists (LABA)). Subgroup analysis was restricted to relapse.

Sensitivity analysis was also carried out by removing the following types of studies from the primary outcome analyses.

1. Studies that were considered to be at high risk of bias based on the criteria outlined in the Cochrane Handbook for Systematic Reviews of Interventions [28].

2. Studies in which the duration of oral corticosteroid treatment was less than five days.

3. The results from fixed-effect models were compared with the random-effects models for the main outcome.

4. Studies in which supplemental corticosteroids were provided to the patients in the ED as a co-intervention.

\section{For Quantitative Synthesis - Adequate Investigation of Publication Bias and Discussion of the Likely Impact on the Results of the Review}

No - the authors did not perform graphical or statistical tests for publication bias and discussed the likelihood and magnitude of the impact of publication bias. The authors recognized that some studies could have been missed. They added that due to an insufficient number of included studies, publication bias could not be assessed as planned. As such, the risk of publication bias was considered unclear.

\section{Did the Review Authors Report any Potential Sources of Conflict of Interest and Funding for Review}

Yes. All internal and external sources of support, as well as funding, how any conflicts of interest were handled were discussed. These sources of funding and support were not involved in the preparation of this protocol and in any aspect of the conduct and reporting of the review. 


\section{AMSTAR 2 Critical Domains}

Seven domains were identified by the developers of AMSTAR 2. A review validity, as well as its conclusions, can be affected by these domains. However, the developers acknowledged that all steps are important.

Applying AMSTAR 2 Critical Domains to Systematic Review Article Selected

1. Protocol registered before the commencement of the review (item 2) - yes.

2. Adequacy of the literature search (item 4) yes.

3. Justification for excluding individual studies (item 7) - yes.

4. Risk of bias from individual studies being included in the review (item 9) - yes.

5. Appropriateness of meta-analytical methods (item 11) - yes.

6. Consideration of risk of bias when interpreting the results of the review (item 13) - yes.

7. Assessment of presence and likely impact of publication bias (item 15) - yes. [23].

\section{Rating the Overall Confidence in the Results of the Review}

There is a proposed method for the interpretation of detected weaknesses in both the non-critical and critical items. The ratings are described as either high/moderate/low/critically low. The full description of the proposed rating scale can be found in supplementary list 3 .

\section{Conclusions}

\section{Article Appraisal Conclusions}

According to the authors, the conclusions are itemized as follows;

1. In determining if IM corticosteroids are more effective in reducing relapse compared to oral corticosteroids among children or adults discharged from an ED or equivalent acute care setting for acute asthma, insufficient evidence was found.

2. Fewer adverse events were observed in patients that received IM corticosteroids. This was, however, not statistically significant.

3. It was suggested that additional studies comparing the effectiveness of IM versus oral corticosteroids could provide further evidence clarity.

4. It was also suggested that further studies should be conducted to compare the different types of IM corticosteroids (i.e., IM triamcinolone, methylprednisone, and dexamethasone), as well as other oral corticosteroids (i.e., oral prednisone and dexamethasone). The dosage and pharmacokinetics of these drugs should also be considered.

5. The preference of patients, as it relates to adherence, should also be considered.

The overall confidence in the above conclusions from this appraisal is rated as high because there was no non - critical weakness in the evaluation of the critical domains of the review. In view of the above, it is not advisable to proceed to EBM steps 4 and 5. The appraisal of results of further studies as enumerated above may engender the full implementation of 5-step EBM as appropriate.

\section{Conflict of Interest}

The author declares that there is no conflict of interest.

\section{Acknowledgement}

Both SPT and SEO formulated the review question, the review topic, literature search, article selection, article critical appraisal, and the write-up of this evidence-based medicine review. 


\section{References}

[1] Croisant S. 2014 Epidemiology of Asthma: Prevalence and Burden of Disease. In: Brasier A. (eds) Heterogeneity in Asthma. Advances in Experimental Medicine and Biology, vol 795:17-29 Humana Press, Boston, MA. https://doi.org/10.1007/978-1-4614-8603-9_2.

[2] Rowe BH, Kirkland SW, Vandermeer B, et al. 2017. Prioritizing Systemic Corticosteroid Treatments to Mitigate Relapse in Adults With Acute Asthma: A Systematic Review and Network Metaanalysis. Academic Emergency Medicine: Official Journal of the Society for Academic Emergency Medicine. Mar;24(3):371-381. DOI: 10.1111/acem.13107. PMID: 27664401.

[3] Services UD of $H$ and $H$. National asthma education program: executive summary. Guidelines for the diagnosis and management of asthma Publication NO. 1991.

[4] Topal E, Gücenmez OA, Harmancı K, Arga M, Derinoz O, Turktas I, 2014. Potential predictors of relapse after treatment of asthma exacerbations in children, Annals of Allergy, Asthma \& Immunology, Volume 112 (4), 361-364, ISSN 1081-1206, https://doi.org/10.1016/j.anai.2014.01.025.

[5] Ducharme FM, Zemek RL, Chalut D, McGillivray D, Noya FJ, Resendes S, Khomenko L, Rouleau R, Zhang X. 2011. Written action plan in pediatric emergency room improves asthma prescribing, adherence, and control. Am J Respir Crit Care Med. 15;183(2):195-203. DOI: 10.1164/rccm.2010010115OC. Epub 2010 Aug 27. PMID: 20802165.

[6] McNamara RM, Rubin JM. 1993. Intramuscular methylprednisolone acetate for the prevention of relapse in acute asthma. Ann Emerg Med. 22(12):1829-35. DOI: $\quad$ 10.1016/s01960644(05)80409-3. PMID: 8239103.

[7] Hoffman IB, Fiel SB. 1988. Oral vs. repository corticosteroid therapy in acute asthma. Chest. 93(1):11-3. DOI: 10.1378/chest.93.1.11. PMID: 3275525.

[8] Lee CH, Lee CJ, Lan RS, Tsai YH, Chiang YC, Wang WJ, Tsao TC. 1993. Repository dexamethasone in the treatment of acute bronchial asthma. Changgeng Yi Xue Za Zhi. 16(1):25-9. PMID: 8490772.
[9] Schuckman H, DeJulius DP, Blanda M, Gerson LW, DeJulius AJ, Rajaratnam M. 1998. Comparison of intramuscular triamcinolone and oral prednisone in the outpatient treatment of acute asthma: a randomized controlled trial. Ann Emerg Med. 31(3):333-338. DOI: 10.1016/s0196-0644(98)703439. Erratum in: Ann Emerg Med 1998 Jun;31(6):795. PMID: 9506490.

[10]Chan JS, Cowie RL, Lazarenko GC, Little C, Scott S, Ford GT. 2001. Comparison of intramuscular betamethasone and oral prednisone in the prevention of relapse of acute asthma. Can Respir J. 8(3):147-52. DOI: 10.1155/2001/613426. PMID: 11420590.

[11] Saunders CE. 1987. Patient compliance in filling prescriptions after discharge from the emergency department. Am J Emerg Med.;5(4):283-6. DOI: 10.1016/0735-6757(87)90351-2. PMID: 3297080.

[12] Thomas EJ, Burstin HR, O’Neil AC, Orav EJ, Brennan TA. 1996. Patient noncompliance with medical advice after the emergency department visit. Ann Emerg Med. 27(1):49-55. DOI: 10.1016/s01960644(96)70296-2. PMID: 8572448.

[13]Titler M. Developing an evidence-based practice. 2006.

[14] Sackett DL. Evidence-based medicine: how to practice and teach. EBM. 2000.

[15] Sackett DL, Rosenberg WM, Gray JM, Haynes RB, Richardson WS. 1996. Evidence-based medicine. BMJ: British Medical Journal. 313: 170. DOI: https://doi.org/10.1136/bmj.312.7023.71.

[16]Akobeng AK. Principles of evidence-based medicine. Arch Dis Child. 2005 Aug;90(8):837-40. DOI: 10.1136/adc.2005.071761. PMID: 16040884; PMCID: PMC1720507.

[17] Antman EM, Lau J, Kupelnick B, Mosteller F, Chalmers TC. 1992. A comparison of results of metaanalyses of randomized control trials and recommendations of clinical experts. Treatments for myocardial infarction. JAMA. 1992 8;268(2):240-8. PMID: 1535110.

[18] Oxman AD, Guyatt GH. The science of reviewing research. Ann N Y Acad Sci. 1993 Dec 31;703:125-33; discussion 133-4. DOI: 10.1111/j.1749-6632.1993.tb26342.x. PMID: 8192290. 
[19]Liberati A, Altman DG, Tetzlaff J, Mulrow C, Gøtzsche PC, Ioannidis JP, Clarke M, Devereaux PJ, Kleijnen J, Moher D. 2009. The PRISMA statement for reporting systematic reviews and meta-analyses of studies that evaluate health care interventions: explanation and elaboration. $\mathbf{J}$ Clin Epidemiol. ;62(10):e1-34. DOI: 10.1016/j.jclinepi.PMID: 19631507.

[20] Akobeng AK. 2005. Understanding randomised controlled trials. Arch Dis Child. 90(8):840-4. DOI: 10.1136/adc.2004.058222. PMID: 16040885; PMCID: PMC1720509.

[21] Kang H. 2016. How to understand and conduct evidence-based medicine. Korean J Anesthesiol. 2016 Oct;69(5):435-445.

DOI: 10.4097/kjae.2016.69.5.435. Epub 2016 Sep 8. PMID: 27703623; PMCID: PMC5047978.

[22] Kirkland S, Cross E, Campbell S, Villa-Roel C, Rowe B. 2018. Intramuscular versus oral corticosteroids to reduce relapses following discharge from the emergency department for acute asthma. Cochrane Database of Systematic Reviews.. doi:10.1002/14651858.CD012629.pub2.

[23] Shea BJ, Reeves BC, Wells G, Thuku M, Hamel C, Moran J, et al. AMSTAR 2: a critical appraisal tool for systematic reviews that include randomised or non-randomised studies of healthcare interventions, or both. BMJ. 2017;358: j4008. doi:10.1136/bmj.j4008.

[24] Shea BJ, Grimshaw JM, Wells GA, Boers M, Andersson N, Hamel C, et al. Development of
AMSTAR: a measurement tool to assess the methodological quality of systematic reviews. BMC Medical Research Methodology. 2007;7: 10. doi:10.1186/1471-2288-7-10.

[25] Moher D, Liberati A, Tetzlaff J, Altman DG. Preferred reporting items for systematic reviews and meta-analyses: the PRISMA statement. BMJ. 2009;339: b2535. doi:10.1136/bmj.b2535.

[26] Shea BJ, Hamel C, Wells GA, Bouter LM, Kristjansson E, Grimshaw $\mathbf{J}$, et al. AMSTAR is a reliable and valid measurement tool to assess the methodological quality of systematic reviews. Journal of Clinical Epidemiology. 2009;62: 10131020. doi:10.1016/j.jclinepi.2008.10.009.

[27] Stroup DF, Berlin JA, Morton SC, Olkin I, Williamson GD, Rennie D, et al. Meta-analysis of Observational Studies in EpidemiologyA Proposal for Reporting. JAMA. 2000;283: 2008-2012. doi:10.1001/jama.283.15.2008.

[28]Higgins J, Altman D, Sterne J. Chapter 12: Assessing the risk of bias in included studies. In: Cochrane Handbook for Systematic Reviews of Interventions: The Cochrane Collaboration. 2011. Available: http://handbook.cochrane.org.

[29] Higgins J, Altman D, Sterne J. Chapter 8: Assessing the risk of bias in included studies. In: Cochrane Handbook for Systematic Reviews of Interventions: The Cochrane Collaboration. 2011. Available: http://handbook.cochrane.org. 